Submitted to Acta Materialia in May 2015, Revised in August 2015

\title{
Multiple Ferroic Glasses Via Ordering
}

\author{
J.A. Monroe ${ }^{1}$, J.E. Raymond ${ }^{2}$, X. Xu ${ }^{3}$, M. Nagasako ${ }^{3}$, R. Kainuma ${ }^{3}$, Y.I. Chumlyakov ${ }^{4}$, R. \\ Arroyave $^{1}$, I. Karaman ${ }^{1}$. \\ ${ }^{1}$ Department of Materials Science and Engineering, Texas A\&M University, College Station, TX, USA \\ ${ }^{2}$ Texas A\&M University, Department of Chemistry, College Station, TX, USA \\ ${ }^{3}$ Tohoku University, Department of Materials Science and Engineering, Sendai, Miyagi, Japan \\ ${ }^{4}$ Siberian Physical Technical Institute, Tomsk, Russia \\ *Correspondence to: ikaraman@tamu.edu
}

\begin{abstract}
Structural glasses are characterized by the loss of long-range translational and rotational symmetry. In the last two decades, however, it has been discovered that materials that exhibit ferroic (ferromagnetic, ferroelectric and ferroelastic) phase transformations may also exhibit glassy behavior, in which the ferroic degrees of freedom (magnetization, polarization, strain) exhibit a loss of long-range translational symmetry. A consequence of this loss of longrange symmetry is the suppression of the ferroic phase transitions. Moreover, these unique glassy systems exhibit dynamic and thermodynamic behavior analogous to regular structural glasses. Conventionally, the onset of glassy behavior is brought about by the introduction of spatial heterogeneities, typically originating from point defects, particularly in the case of strain glasses. Here, we demonstrate, for the first time, that configurational order/disorder in a single ferromagnetic alloy $\left(\mathrm{Ni}_{45} \mathrm{Co}_{5} \mathrm{Mn}_{36.6} \operatorname{In}_{13.4}\right)$ can be used to stabilize both strain and magnetic glasses. The control of the degree of configurational order-through simple heat treatment schedules-, and simultaneous application of stress and magnetic field enabled us to observe a Kauzmman point, that is, the collapse of the entropy difference between a crystalline and a glassy phase. Systematic investigation of the transformation behavior in this system as a function of heat treatment enabled us to observe four kinds of solid-solid phase transitions (ferromagnetic-paramagnetic, martensitic transformation, strain and magnetic glass) in a single composition. The alloy investigated can be used to further elucidate the nature of ferroic glass transitions and their coupling.
\end{abstract}

Keywords: Ferroic glasses; strain glass; martensitic transformation; shape memory alloys; magnetic glass. 


\section{Introduction}

\subsection{Overview}

To understand the similarities between conventional (structural), magnetic and strain glasses, it is instructive to first examine glass-forming silica as an example [1]. Figure 1A schematically shows a pure molten silicate undergoing an isothermal phase transformation from an amorphous liquid to a symmetric crystal at its melting point $\left(\mathrm{T}_{\mathrm{m}}\right)$. However, the amorphous structure can be stabilized at slow cooling rates by introducing $\mathrm{Na}$ defects that frustrate the erystal's long range crystalline translational/rotational symmetry. This allows the liquid's amorphous liquid structure to be super-cooled past $\mathrm{T}_{\mathrm{m}}$ producing a second order transition $\left(\mathrm{T}^{*}\right)$ and frozen below the second-order glass transition temperature $\left(\mathrm{T}_{\mathrm{g}}\right)$. The unfrozen glass's glassy relaxation dynamics follow a Vogel-Fulcher-Tamman type behavior between $\mathrm{T}^{*}$ and $\mathrm{T}_{\mathrm{g}}$, while the frozen glass's glassy relaxation dynamics follow an Arrhenius type behavior below $\mathrm{T}_{\mathrm{g}}$. The critical transitions and the resulting relaxation dynamics associated with them are used to identify and characterize the behavior in conventional glassy phases. Similar relaxation dynamics and transitions have been used to describe ferroic glasses [2-7] even though the transition is between two crystalline solids instead of an amorphous liquid and crystalline solid.

The schematic of entropy vs temperature response of a strain glass is shown in Figure 1B in the alloys under study, as an example, which shows thermo-elastic martensitic transformation. As the high temperature austenite phase is cooled, a first-order phase transformation to martensite occurs at the martensite start temperature $\left(\mathrm{T}_{\mathrm{Ms}}\right)$. This transformation is characterized by diffusionless atomic displacements, resulting in a spontaneous breaking of the symmetry of the high temperature phase. According to conventional theories about the strain glass transition, once a critical concentration of point defects is present, the martensitic transformation is replaced by a supercooling transition at $\mathrm{T}^{*}$ followed by a second order strain glass freezing at $\mathrm{T}_{\mathrm{g}}$. Current understanding of strain glass behavior suggests that defects cause local random lattice distortions along the austenite-to-martensite deformation path. The resulting random planar spacing along preferred crystallographic directions produces an anisotropic glassy structure that frustrates the long-range martensitic transformation strain [8]. In other words, the atoms in the anisotropic glass have translational symmetry along all directions (like a traditional crystal) except along the austenite to martensite deformation pathway where a non-periodic amorphous structure exists (like a traditional glass). Interestingly, the resulting strain glass can exhibit superelasticity 
between $T^{*}$ and $T_{g}$ and shape memory effect below $T_{g}$ [4]. To date, evidence for strain glass transition has been found in off-stoichiometric NiTi(Fe,Co, $\mathrm{Cr}, \mathrm{Mn}$ ), $\mathrm{NiCoMnGa}$, and $\mathrm{NiCoFeGa}$, $\mathrm{NiMnIn}(\mathrm{Fe}, \mathrm{Cr}), \mathrm{CeFe},(\mathrm{La}, \mathrm{Pr}, \mathrm{Ca}) \mathrm{MnO}_{3}$ alloys. However, there is still no conclusive evidence as to the nature of the point defects responsible for the onset of strain glass behavior [2,4-11].

The same analogy with conventional structural glasses can be extended to magnetic glasses as displayed in Figure 1C [12]. An ordinary paramagnetic to ferromagnetic transition is characterized by magnetic spin alignment along a single direction that creates long-range translational/rotational magnetic symmetry. In contrast to the martensitic transformation, the ferromagnetic transition is second-order and strengthens during cooling below the Curie temperature $\left(\mathrm{T}_{\mathrm{C}}\right)$. If sufficient disorder exists in a ferromagnetic system (due to point defects or due to competing ferro and anti-ferro magnetic interactions), spins are prevented from arranging themselves in patterns with long-range symmetry. The 'amorphous' magnetic structure is supercooled below $T^{*}$ and then freezes below a critical temperature $\left(T_{f}\right)$. These spin glasses were first discovered by doping $\mathrm{Cu}$ with $\mathrm{Mn}$ magnetic point defects, although in this case the magnetic spins are isolated and only interact weakly with each other [13]. In contrast, in some other magnetic glasses the magnetic spins are arranged into clusters that have local ferromagnetic ordering, but do not form long-range symmetric structures with each other [14,15]; a structural glass analogy is a semi-crystalline material. Evidence of spin and cluster spin glasses have been observed in GdGe, the low temperature martensite phase of CuZnAlMn shape memory alloys (SMAs) and NiCoMnSn magnetic SMAs (MSMAs) [14-18].

Another type of glass, not shown in Figure 1, similar to the strain and magnetic glasses described above can be observed when electric dipole alignment inside a material does not have long-range translational or rotational symmetry. These ferroelectric glasses are commonly referred to as relaxor ferroelectrics and share similar Vogel-Fulcher-Tamman and Arrhenius relaxation in their unfrozen and frozen disordered states, respectively, with the glasses described above $[19,20]$. Relaxor ferroelectric glasses have been observed simultaneously with strain glasses in materials such as $\mathrm{PbLa}(\mathrm{ZrTi}) \mathrm{O}$ (PLZT) perovskites where a martensitic transformation is coupled to a polarization [20].

A common phenomenon described as "tweed" or cross-hatched microstructures have been observed in materials that undergo thermo-elastic martensitic transformations. These structures 


\subsection{The Kauzmann Paradox}

Although the frustrated microscopic degrees of freedom are different from one another, the different kinds of glasses described above can be treated in a similar thermodynamic manner at macroscopic scales. One similarity is the extrapolated entropy of the high temperature phase intersecting the entropy curve of the low temperature phase in Figure 1A-1C. At this critical temperature, known as the Kauzmann point $\left(\mathrm{T}_{\mathrm{K}}\right)$, the entropies of the high and low temperature phases become identical $[23,24]$. This critical point is considered a paradox in the case of structural glasses because it is was deemed impossible for a frozen amorphous phase, with high configurational entropy contributions from disorder, to have lower entropy than a highly ordered crystalline phase [24].

Due to a glass' tendency to undergo a glass transition before the Kauzmann point is reached, $\mathrm{T}_{\mathrm{K}}$ has only been directly observed in special cases such as polymer chains with very small breadth-distribution and Helium III and IV [24]. Figure 2 displays a schematic diagram for Helium III or IV adapted from [24] where direct observation of a Kauzmann point occurs in the pressure-temperature phase diagram. The slope of the first order phase transformation line can be used to determine the relative entropy of the two phases by using the Clausius-Clapeyron relationship $\frac{d P}{d T}=\frac{\Delta S}{\Delta V}$. The volume of the liquid phase is assumed to remain greater than the crystalline phase, which makes the $\Delta V$ term negative. At high temperatures, the $\frac{d P}{d T}$ slope is positive which indicates that $\Delta S$ must be negative and the entropy of the crystalline phase is less 
than the liquid phase. Similarly, below $\mathrm{T}_{\mathrm{K}}, \frac{d P}{d T}$ becomes negative which indicates that $\Delta S$ is positive and the liquid phase has lower entropy than the crystalline phase. The point at which the slope changes from positive to negative is defined as $T_{K}$ and has been calculated to be $\sim 1 / 2 T_{m}$ for many pure metallic elements $[19,25]$. To prevent violation of the third law of thermodynamics, another Kauzmann point must exist as the system approaches absolute zero, i.e. the $\frac{d P}{d T}$ slope approaches zero as $T \rightarrow 0$ [24]. The Clausius-Clapeyron relationship for He III and He IV represents a hysteresis-free first order transition and care must be used when extending this analysis to the first order transition with hysteresis found in SMAs and MMSMAs.

\subsection{Alloy Selection}

To date, the strategy to stabilize a strain glass in materials that show first order martensitic transformation has been to add impurities that suppress the martensitic transformation and promote the formation of a glassy state beyond a critical concentration [2,6-9]. In the present work, the same strain glass stabilization is shown to occur by manipulating the degree of configurational order and defect clusters in a single alloy composition. Control of configurational order also leads to the onset of cluster spin glass stabilization in the material's martensitic phase of the material. By holding the composition constant in the alloy under investigation, i.e. $\mathrm{Ni}_{45} \mathrm{Co}_{5} \mathrm{Mn}_{36.6} \mathrm{In}_{13.4}$ magnetic shape memory alloy (MSMA), the strain glass / cluster spin glass is examined in this work without affecting the alloy's electron-to-atom ratio (e/a ratio) or magnetic valence that have considerable influence on its properties [26-28]. Different degrees of configurational order, which produce different defect concentrations and distributions, were achieved in this off-stoichiometric Heusler alloy through heat treatments between $673 \mathrm{~K}$ and $1073 \mathrm{~K}$. It is will be shown here in the present work that these heat treatments affect the material's configurational order and point defect concentration of the material which enhances $\mathrm{T}_{\mathrm{C}}$ and $\mathrm{T}_{\mathrm{f}}$, suppresses $\mathrm{T}_{\mathrm{Ms}}$ and ultimately stabilizes the formation of a strain glass phase.

NiCoMnIn was selected because it has been studied extensively in the MSMA literature, can be easily grown into single crystals, and is expected to exhibit responses representative of the materials that show strain or spin glasses by compositional control [29-37]. Single crystals were used to eliminate grain boundary effects. The B2 ordered crystals were subjected to secondary 
heat treatments between $673 \mathrm{~K}$ and $1073 \mathrm{~K}$ for three hours and then water quenched (WQ) to increase the $\mathrm{L} 22_{1}$ ordering. The impact of $\mathrm{B} 2$ vs $\mathrm{L} 2_{1}$ ordering on the martensitic and ferromagnetic transitions, as well as on the onset of the ferroic glass transitions were investigated. The present work differs from the previous ones that utilizes changes in chemical composition to observe traditional and ferroic glasses [2, 4-11,13-18].

\section{Experimental Procedures}

Elemental $\mathrm{Ni}, \mathrm{Co}, \mathrm{Mn}$ and In were induction melted and pre-cast into a copper chilled mold with a nominal $\mathrm{Ni}_{45} \mathrm{Co}_{5} \mathrm{Mn}_{36.6} \mathrm{In}_{13.4}$ composition. The single crystals were then grown from these ingots in a He environment using the Bridgman technique. Single crystal samples were wire electro-discharge machined to $2 \times 2 \times 4 \mathrm{~mm}^{3}$ for uniaxial compression testing and $0.5 \times 2 \times 26 \mathrm{~mm}^{3}$ for dynamic mechanical analysis (DMA) in single cantilever beam mode. The long axes for uniaxial compression and for DMA testing were cut along the [100] crystallographic orientation. The crystals were sealed in quartz ampoules and homogenized at $1173 \mathrm{~K}$ for 24 hours, then water quenched (WQ). They were then sealed under argon again in quartz ampoules and subjected to secondary heat treatments between $673 \mathrm{~K}$ and $1073 \mathrm{~K}$ for 3 hours then WQ prior to magnetothermo-mechanical testing. Samples for Transmission Electron Microscopy (TEM) were cut from single crystals with the (110) direction perpendicular to the sample surface and twin jet electro polished.

DC magnetic properties were measured using a Quantum Design Superconducting Quantum Interference Device - Vibrating Squid Module (SQUID-VSM) and AC magnetic properties were measured using a traditional extraction style SQUID magnetometer with an AC magnetic coil. For the DC experiments, all samples were cooled to $5 \mathrm{~K}$ under zero magnetic field, a $0.05 \mathrm{~T}$ biased field was applied and the sample heated and cooled between $5 \mathrm{~K}$ and $400 \mathrm{~K}$. The first heating is referred to as the zero field cooled (ZFC) response while the cooling under $0.05 \mathrm{~T}$ and the subsequent heating under the same field is referred to as the field cooled (FC) response. For $\mathrm{AC}$ magnetic measurements, the real part of the magnetization was measured every $1^{\circ} \mathrm{C}$ in a $30^{\circ} \mathrm{C}$ temperature window, spanning the cluster spin glass freezing temperature, using $0.05 \mathrm{~T}$ amplitude AC magnetic excitation frequencies logarithmically spaced between $1 \mathrm{~Hz}$ and $1000 \mathrm{~Hz}$. 
The magnetization and strain vs. magnetic field responses under constant uniaxial compression were characterized using a custom built micro-magneto-thermo-mechanical test frame (micro MTM) [35]. The micro MTM's body and inner components of the micro MTM are made of from precipitation hardened nonmagnetic $\mathrm{Cu}-\mathrm{Be}$ and can apply compressive stresses on the specimens using 316L stainless steel Belleville springs driven by a screw mechanism. The Belleville springs were specially designed such that the change in applied stress due to thermal expansion during heating/cooling experiments in the temperature range of $300 \mathrm{~K}$ to $4.2 \mathrm{~K}$ is not more than $\pm 3 \mathrm{MPa}$ for the given sample dimensions. Displacements with an accuracy of \pm 0.0001 $\mathrm{mm}$ are measured using a miniature capacitive sensor that is capable of measuring the displacement at temperatures as low as $4.2 \mathrm{~K}$ and magnetic fields as high as 18 Tesla. The micro MTM was placed in an extraction type $18 \mathrm{~T}$ magnet to obtain magnetization and strain measurements on the specimens under magnetic fields from 0 to 18 Tesla, test temperatures ranging from 4.2 to $300 \mathrm{~K}$ and stress levels up to $125 \mathrm{MPa}$.

Using the micro-MTM, the samples were loaded to $-80 \mathrm{MPa}$ at room temperature, cooled below the martensitic transformation temperature then heated to the desired temperature for magnetization testing. Calibration of the load cell verified that the $-80 \mathrm{MPa}$ stress was held constant between $\pm 5 \mathrm{MPa}$ during temperature sweeps and phase transformation. The magnetic field was ramped to $18 \mathrm{~T}$ at a rate of $0.3 \mathrm{~T}$ per minute.

DMA testing was conducted in single cantilever beam mode using a Mettler Toledo TTDMA. The sample was cooled to $125 \mathrm{~K}$ under $0 \mathrm{MPa}$ then heated at $5 \mathrm{~K}$ per minute to $400 \mathrm{~K}$ while measuring the logarithmically spaced frequency dependent elastic modulus (E') between $0.2 \mathrm{~Hz}$ and $25 \mathrm{~Hz}$. The compliance modulus between logarithmically spaced $0.05 \mathrm{~Hz}$ and $6.3 \mathrm{~Hz}$ was measured at $150 \mathrm{~K}, 175 \mathrm{~K}, 200 \mathrm{~K}$ and $225 \mathrm{~K}$ to determine the characteristic relaxation time $\left(\tau_{0}\right)$ at each temperature.

\section{Experimental Results}

\subsection{Magnetic and Martensitic Transitions}

Magnetization vs. temperature plots for samples that underwent martensitic transformation were used to determine the spin glass freezing $\left(T_{f}\right)$, martensite start $\left(T_{M s}\right)$ and Curie $\left(T_{C}\right)$ temperatures. as shown in Figure 3A displays an example of these transformations for the $873 \mathrm{~K}$ 


\subsection{Strain Glass Transitions}

In the case of the $673 \mathrm{~K}-3 \mathrm{hr}-\mathrm{WQ}$ samples, the thermally induced martensitic transformation was completely suppressed. No sign of martensitic transformation was detected either with differential scanning calorimetry down to $120 \mathrm{~K}$ or SQUID down to $6 \mathrm{~K}$. The $673 \mathrm{~K}-$ 3hr - WQ sample condition was also subjected to the dynamic mechanical analysis (DMA) displayed in Figure 4 to characterize its relaxation dynamics. Above $300 \mathrm{~K}$ the storage modulus 


\subsection{Kauzmann Point}

To determine $\mathrm{T}_{\mathrm{K}}$ for the $673 \mathrm{~K}-3 \mathrm{hr}-\mathrm{WQ}$ condition, the martensitic transformation was first stabilized with stress (-80 MPa) and the critical magnetic fields for martensitic transformation were then determined at various temperatures. In other words, even though the material do not exhibit thermally-induced martensitic transformation, it is possible to induce the transformation with external stress. The -80 MPa load-stress was sufficient to stabilize the martensite upon cooling, in a similar manner to stress induced crystallization in amorphous polymers. Once the martensite is stabilized below $\mathrm{T}^{*}$ (Figure 4), the critical magnetic field levels to trigger martensite to austenite reverse transformation were determined at various temperatures. The magnetization (solid) and strain (dashed) vs magnetic field response for the [100] oriented compression sample under $-80 \mathrm{MPa}$ is displayed in Figure 5. Following the $150 \mathrm{~K}$ test, the sample begins in the martensitic state at $0 \mathrm{~T}$. The martensite is quickly magnetized to a low saturation point and the strain remains fairly constant as the magnetic field is ramped between 0 

transformation from martensite to austenite occurs as shown by the large increase in magnetization and decrease in transformation strain. The transformation is completed around $5 \mathrm{~T}$ as shown by the magnetization and strain saturation between $5 \mathrm{~T}$ and $10 \mathrm{~T}$. As the magnetic field is removed, the strain and magnetization remain fairly constant and retraces the magnetic loading path until the austenite to martensite transformation occurs between $2.5 \mathrm{~T}$ and $0.5 \mathrm{~T}$. The critical fields required for martensitic transformation were determined using the slope intercept method shown in the $110 \mathrm{~K}$ curves. As the testing temperature decreases from $190 \mathrm{~K}$ to $30 \mathrm{~K}$, the critical magnetic field required for the martensite to austenite transformation increases while the critical field for austenite to martensite transformation first increases then decreases. These experiments also show that the magnetic saturation of the martensite phase remains lower than the magnetic saturation of the austenite phase down to $30 \mathrm{~K}$.

Figure 6 displays the temperature dependent critical magnetic fields for the martensite start $\left(\mathrm{H}_{\mathrm{Ms}}\right)$ and austenite finish $\left(\mathrm{H}_{\mathrm{Af}}\right)$ magnetic fields determined from the magnetization and strain measurements given in Figure 5. The equilibrium magnetic field $\left(\mathrm{H}_{0}\right)$, defined as the average of the forward and reverse transformation critical fields, is plotted as well. This equilibrium magnetic field is considered to be the equilibrium point in the critical field - temperature phase diagram. Calculating $\mathrm{H}_{0}$ using this averaging technique has been shown to be rate-independent in NiCoMnIn when comparing slowly ramped and rapidly pulsed magnetic field induced phase transformations [36].

Using the analysis scheme described for Figure 2, a Kauzmann point at $80 \mathrm{~K}$ is observed. Here, the temperature dependent slope of the equilibrium magnetic field for phase transformation is used to determine the relative entropy of the two phases by applying the Clausius-Clapeyron relationship $\frac{d H_{0}}{d T}=\frac{-\Delta S^{A \rightarrow M}}{\Delta M^{A \rightarrow M}}$; where $\Delta M^{A \rightarrow M}=M^{M}-M^{A}$ and $\Delta S^{A \rightarrow M}=S^{M}-S^{A}$ are the differences in magnetization and entropy, respectively, between martensite and austenite. It should be noted that although the strain is changing while ramping the magnetic field, the stress remains constant and therefore does not appear in this Clausius-Clapeyron relationship. The martensite magnetization remains lower than austenite magnetization, evident in the magnetization curves in Figure 5, which makes the $\Delta M^{A \rightarrow M}$ term negative throughout the experimental temperature range. Above $80 \mathrm{~K}$, the $\frac{d H_{0}}{d T}$ slope is negative indicating that the 


\section{Discussion of the Results}

\subsection{The Role of Ordering Heat Treatments}

Secondary heat treatments on NiMn based MMSMAs between $673 \mathrm{~K}$ and $1073 \mathrm{~K}$ change the atomic order [40] and in the present study we show that these heat treatments in turn change all the other material transitions. The $\mathrm{B} 2$ or $\mathrm{CsCl}$ structure displayed in Figure 7A is created when one atom type, $\mathrm{Ni}$ and $\mathrm{Co}$ in this case, occupy the body centered lattice location and the other lattice sites are another atom type, either Mn or In. This is the ordered structure obtained from quenching the homogenized sample from sufficiently high temperatures. If the sample is held below the B2-to-L2 1 transition temperature, further ordering to the structure, displayed in Figure 7B, occurs as the Mn and In atoms settle into separate lattice locations. To obtain a complete L2 ${ }_{1}$ ordered system (Figure 7), a stoichiometric composition of $(\mathrm{Ni} / \mathrm{Co})_{2} \mathrm{MnIn}$ is required in the NiCoMnIn alloy. While the selected $\mathrm{Ni}_{45} \mathrm{Co}_{5} \mathrm{Mn}_{36.6} \mathrm{In}_{13.4}$ composition is off this stoichiometry, L2 1 ordering still occurs below $\sim 896 \mathrm{~K}$ and its morphology can be varied by varying the time and temperature of secondary heat treatments [30]. Figure 8 displays dark field TEM images of the austenitic L2 1 characteristic (111) superlattice diffraction spot for the samples heat treated between $673 \mathrm{~K}$ and $1073 \mathrm{~K}$ for 3 hours. The bright areas correspond to L2 1 ordering while the dark areas are B2 ordered. The L2 1 morphology is $\sim 10 \mathrm{~nm}$ or less in size for Figure $8 \mathrm{~d}-973 \mathrm{~K}$, Figure 8e - $1073 \mathrm{~K}$ and Figure $8 \mathrm{f}-1173 \mathrm{~K}$ heat treated samples. The $873 \mathrm{~K}$ heat treated sample displayed in Figure 8c contains larger sized L2 $2_{1}$ regions that are less uniform while the $773 \mathrm{~K}$ heat treated sample displayed in Figure $8 \mathrm{~b}$ exhibits $100-150 \mathrm{~nm}$ rectangular $\mathrm{L} 2_{1}$ regions. The fully arrested $673 \mathrm{~K}$ sample displayed in Figure 8a exhibits a uniform distribution of L2 ${ }_{1}$ regions that are $15 \mathrm{~nm}$ or less. These $\mathrm{B} 2-\mathrm{L} 22_{1}$ ordering boundaries can be varied without changing the 
composition and are thought to be the source of the strain and cluster spin glasses. More systematic studies must be conducted to determine the exact defect structures that create the strain glass phenomenon.

\subsection{Phase Diagram}

The extracted $\mathrm{T}_{\mathrm{C}}, \mathrm{T}_{\mathrm{Ms}}, \mathrm{T}_{\mathrm{f}}, \mathrm{T}_{\mathrm{g}}, \mathrm{T}^{*}$ and $\mathrm{T}_{\mathrm{K}}$ critical transitions for all the samples heat treated between $673 \mathrm{~K}$ and $1073 \mathrm{~K}$ have been assembled into the phase diagram displayed in Figure 9. The heat treatments between $773 \mathrm{~K}$ and $1073 \mathrm{~K}$ lead to a transformation sequence similar to the response displayed in Figure 4. In this region of Figure 9, $\mathrm{T}_{\mathrm{C}}$ and $\mathrm{T}_{\mathrm{f}}$ increase linearly while $\mathrm{T}_{\mathrm{Ms}}$ decreases linearly with decreasing heat treatment. The samples heat treated between $673 \mathrm{~K}$ and $773 \mathrm{~K}$ also respond similarly to the sample in Figure 5, but $\mathrm{T}_{\mathrm{f}}$ increases exponentially while $\mathrm{T}_{\mathrm{C}}$ continues to increase linearly. $\mathrm{T}_{\mathrm{Ms}}$ first decreases exponentially then rises rapidly indicating unstable supercooling in the gold triangular phase field. The $673 \mathrm{~K}$ heat treatment has a very different response that originates from the absence of a martensitic transformation under $0 \mathrm{MPa}$. Instead of $\mathrm{T}_{\mathrm{Ms}}$, a super cooling transition at $\mathrm{T}^{*}$ that falls along the linear extrapolation of $\mathrm{T}_{\mathrm{Ms}}$. This gives evidence to $\mathrm{T}^{*}$ originating from a transition between stable austenite and an unfrozen strain glass at the extrapolated $\mathrm{T}_{\mathrm{Ms}}$ point. The unfrozen strain glass is then expected to freeze below $\mathrm{T}_{\mathrm{g}}$. The $\mathrm{T}_{\mathrm{M}_{\mathrm{s}}}^{-80 \mathrm{MPa}}$ value obtained during cooling under $-80 \mathrm{MPa}$, obtained from the experiments in Figure 6, displays a continued exponential trend in $\mathrm{T}_{\mathrm{Ms}}$ allowing completion of the unstable supercooled phase field (gold triangle). While $\mathrm{T}_{\mathrm{K}}^{-80 \mathrm{MPa}}$ falls below $\mathrm{T}_{\mathrm{g}}$, as expected from the relationship between $\mathrm{T}_{\mathrm{K}}$ and $\mathrm{T}_{\mathrm{g}}$ in Figure 1B, the 0MPa Kauzmann point may be above $\mathrm{T}_{\mathrm{g}}$.

\subsection{Magnetic Transitions}

Due to the oscillatory nature - ferromagnetic-to-antiferromagnetic —of the Mn-Mn magnetic exchange constants (Ruderman-Kittel-Kasuya-Yosida (RKKY) exchange [41-43]), re-arranging $\mathrm{Mn}$ atoms as the alloy goes from B2 to $\mathrm{L} 2{ }_{1}$ ordering, the degree of configurational order is expected to influence the magnetic properties of Mn-containing Heusler alloys. The increases in $\mathrm{T}_{\mathrm{C}}$ between the $1073 \mathrm{~K}$ and $673 \mathrm{~K}$ heat treatments suggest greater volume fraction of $\mathrm{L} 2{ }_{1}$ 
ordered domains, which is experimentally demonstrated by diffraction experiments on $\mathrm{Ni}_{45} \mathrm{Co}_{5} \mathrm{Mn}_{36.7} \mathrm{In}_{13.3}$ [26]. While the $\mathrm{L} 2_{1}$ ordering occurs in the high temperature austenite phase, the martensite's $T_{f}$ of the martensite is still affected because it inherits the austenite's morphology and defect structures shown in Figure 8. The cluster spin glass in Figure 1C displays a possible ferromagnetic, blue, and antiferromagnetic, yellow, domain structure created by Mn anti-site defects that change the average Mn-Mn distance. It is believed that these magnetic defects stabilize the cluster spin glass causing the increases in $T_{f}$.

\subsection{Martensitic and Strain Glass Transitions}

Decreasing heat treatment temperatures stabilizes the austenite phase and pushes $\mathrm{T}_{\mathrm{Ms}}$ to lower temperatures. The deviation from the linear trend between $773 \mathrm{~K}$ and $673 \mathrm{~K}$ indicates that the austenite undergoes a transition from athermal martensite to isothermal martensite and is easily undercooled. This is also evident in the suppressed $\mathrm{T}_{\mathrm{Ms}}$ for the $673 \mathrm{~K}$ sample under -80 $\mathrm{MPa}$. The stress induced martensitic transformation in this strain glass is analogous to stress induced crystallization in amorphous glasses. The sudden increase in $\mathrm{T}_{\mathrm{Ms}}$ for heat treatments very close to $673 \mathrm{~K}$ shows the complex response of the metastable austenite in the gold colored phase field. The $\mathrm{T}^{*}$ transition determined from DMA falls along the linearly extrapolated $\mathrm{T}_{\mathrm{Ms}}$ curve which strongly indicates that $\mathrm{T}^{*}$ is where the austenite becomes supercooled into an unfrozen strain glass. It is believed that the defects responsible for the anisotropic, or strain, glass are vacancies, anti-site defects and the L2 $2_{1}$ morphology shown in Figure 8. Similar defect structures are expected to cause the mirrored exponential decrease and increase in $T_{M s}$ and $T_{f}$, respectively. This is captured in the anti-site defect illustrations in Figure 1B and 1C.

\subsection{Thermodynamic Arrest}

The three external fields (stress, magnetic field and temperature) necessary to stabilize the phase transformation were used to determine the thermodynamic arrest temperature, $T_{K}$. Although they occur close to one another, $\mathrm{T}_{\mathrm{g}}$ and $\mathrm{T}_{\mathrm{K}}$ should not be co-dependent in the present alloys. Indications of $\mathrm{T}_{\mathrm{K}}$ around $150 \mathrm{~K}$ have been observed in the $\frac{d H_{0}}{d T}$ response of MSMAs that do not undergo a strain glass transition [29, 31, 37, 44]. It has been reasoned that entropy change 


\section{Summary and Conclusions}

To conclude, in this work it has been shown how the control of B2/L2 $2_{1}$ ordering can lead to the onset of strain and magnetic glass transitions in a single Ni-Co-Mn-In off-stoichiometric Heusler alloy composition. We show that with simple heat treatments that ultimately result in different fractions of $\mathrm{L} 22_{1}$ vs B2 ordering in the microstructure, it is possible to control the onset of four solid-solid phase transitions (paramagnetic-ferromagnetic, austenite-martensite, strain and magnetic glasses). The observed dynamical behavior of the strain and spin glass states of the alloy are consistent with the dynamics expected from glassy phases. Interestingly, the coupling between magnetic and strain degrees of freedom enabled us to observe a Kauzmann point where the entropy difference between martensite and austenite collapse. These materials provide an 
excellent model for studying this phenomenon as direct observation of the Kauzmann point is extremely rare in glassy systems. Future work on these types of multi-ferroic glass forming systems should focus on identifying the defect structures responsible for the glassy behavior in these materials in order to allow suppression or enhancement of this glassiness via compositional variations and thermal treatments. Mapping the dependence of the Kauzmann point's dependence on the three intensive variables; temperature, stress and magnetic field, should also be investigated. These endeavors would bring greater insights into the nature of ferroic glass transitions in particular and general glassy phases in general.

\section{Acknowledgments}

The present work was supported by U.S. National Science Foundation (NSF), Division of Materials Research, Metals and Metallic Nanostructures Program, Grant No. 1508634, and NSF - U.S. National Science Foundation - International Materials Institutes Program, grant no. DMR 08-44082. The Japan part was supported by Grant-in-Aids from the Japanese Society for the Promotion of Science (JSPS), and by the Global COE Program, Tohoku University, MEXT, Japan. YIC acknowledges the financial support from RFBR. JAM thanks Dr. Andrey Prosvirin for his help is collecting the AC-SQUID data and the NSF for a grant to purchase the ACSQUID magnetometer and the DOE (DE-8208; FG02-8208; **02ER45999) for support of Dr. Prosvirin.

\section{References:}

[1] B. E. Warren, J. Biscoe, Fourier analysis of X-ray patterns of soda-silica glass, J. Am. Ceram. Soci. 1938, 21, 259.

[2] Y. Wang, C. Huang, J. Gao, S. Yang, X. Ding, X. Song, X. Ren, Evidence for ferromagnetic strain glass in Ni-Co-Mn-Ga Heusler alloy system, App. Phys. Lett. 2012, 101, 101913.

[3] Y. Wang, X. Ren, K. Otsuka, A. Saxena, Evidence for broken ergodicity in strain glass, Phys. Rev. B 2007, 76.

[4] Y. Wang, X. Ren, K. Otsuka, A. Saxena, Temperature-stress phase diagram of strain glass Ti48.5Ni51.5, Acta Mater. 2008, 56, 2885. 
[5] J. Zhang, Y. Wang, X. D. Ding, Z. Zhang, Y. M. Zhou, X. B. Ren, D. Wang, Y. C. Ji, M. H. Song, K. Otsuka, J. Sun, Spontaneous strain glass to martensite transition in a Ti50Ni44.5Fe5.5 strain glass, Phys. Rev. B 2011, 84.

[6] Y. M. Zhou, D. Z. Xue, X. D. Ding, K. Otsuka, J. Sun, X. B. Ren, High temperature strain glass in Ti-50(Pd50-xCrx) alloy and the associated shape memory effect and superelasticity, App. Phys. Lett. 2009, 95.

[7] Y. M. Zhou, D. Z. Xue, X. D. Ding, Y. Wang, J. A. Zhang, Z. Zhang, D. Wang, K. Otsuka, J. Sun, X. B. Ren, Strain glass in doped Ti-50(Ni50-xDx) (D = Co, Cr, Mn) alloys: Implication for the generality of strain glass in defect-containing ferroelastic systems, Acta Mater. 2010, 58, 5433.

[8] X. B. Ren, Y. Wang, K. Otsuka, P. Lloveras, T. Castan, M. Porta, A. Planes, A. Saxena, Ferroelastic nanostructures and nanoscale transitions: ferroics with point defects, MRS Bull. 2009, 34, 838.

[9] D. P. Wang, X. Chen, Z. H. Nie, N. Li, Z. L. Wang, Y. Ren, Y. D. Wang, Transition in superelasticity for Ni55-xCoxFe18Ga27 alloys due to strain glass transition, Epl 2012, 98.

[10] V. K. Sharma, M. K. Chattopadhyay, S. K. Nath, K. J. S. Sokhey, R. Kumar, P. Tiwari, S. B. Roy, The effect of substitution of $\mathrm{Mn}$ by $\mathrm{Fe}$ and $\mathrm{Cr}$ on the martensitic transition in the Ni50Mn34In16 alloy, J. Phys.-Condens. Mat. 2010, 22.

[11] K. Kumar, A. K. Pramanik, A. Banerjee, P. Chaddah, S. B. Roy, S. Park, C. L. Zhang, S. W. Cheong, Relating supercooling and glass-like arrest of kinetics for phase separated systems: Doped CeFe2 and (La,Pr,Ca)MnO3, Phys. Rev. B 2006, 73, 184435.

[12] K. Binder, A. P. Young, Spin-glasses - experimental facts, theoretical concepts, and open questions, Rev. Mod. Phys. 1986, 58, 801.

[13] S. F. Edwards, P. W. Anderson, Theory of spin glasses, J. Phys. F Met. Phys. 1975, 5, 965.

[14] D. Y. Cong, S. Roth, L Schultz, Magnetic properties and structural transformations in NiCo-Mn-Sn multifunctional alloys, Acta Mater. 2012, 60, 5335.

[15] K. P. Bhatti, S. El-Khatib, V. Srivastava, R. D. James, C. Leighton, Small-angle neutron scattering study of magnetic ordering and inhomogeneity across the martensitic phase transformation in Ni50-xCoxMn40Sn10 alloys, Phys. Rev. B 2012, 85.

[16] S. B. Roy, M. K. Chattopadhyay, P. Chaddah, J. D. Moore, G. K. Perkins, L. F. Cohen, K. A. Gschneidner, Jr., V. K. Pecharsky, Evidence of a magnetic glass state in the magnetocaloric material Gd5Ge4, Phys. Rev. B 2006, 74, 012403.

[17] M. O. Prado, F. C. Lovey, L. Civale, Magnetic properties of $\mathrm{Cu}-\mathrm{Mn}-\mathrm{Al}$ alloys with shape memory effect Acta Mater. 1998, 46, 137. 
[18] P. K. Yoo, G. S. Jeen, H. Y. Park, H. K. Kim, S. T. Park, H. C. Lee, J. B. Park, Spin glass behaviour of Cu-Zn-Al-Mn shape memory alloy, J. Mater. Sci. Lett. 1998, 17, 173.

[19] T. Lookman, P. Littlewood, Nanoscale Heterogeneity in Functional Materials, MRS Bull. 2009, 34 (11), 822.

[20] Y. Wang, X. B. Ren, K. Otsuka, Strain Glass: Glassy Martensite, Mater. Sci. Forum 2008, $583,67$.

[21] A. Planes, P. Lloveras, T. Castán, A. Saxena, M. Porta, Ginzburg-Landau modelling of precursor nanoscale textures in ferroelastic materials. Continuum Mech. Therm. 2012, 24, 619.

[22] X. Ren, Y. Wang, Y. Zhou, Z. Zhang, D. Wang, G. Fan, K. Otsuka, T. Suzuki, Y. Ji, J. Zhang, Y. Tian, S. Hou, X. Ding, Strain glass in ferroelastic systems: Premartensitic tweed versus strain glass. Philos. Mag. 2010, 90, 141.

[23] R. W. Cahn, Materials science - multiple Kauzmann paradoxes, Nature 1995, 373, 475.

[24] F. H. Stillinger, P. G. Debenedetti, T. M. Truskett, The Kauzmann paradox revisited, J. Phys. Chem. B 2001, 105, 11809.

[25] K. Kishore, H. K. Shobha, Entropy catastrophe and configurational entropies in supercooled and superheated regimes, J. Chem. Phys. 1994, 101, 7037.

[26] T. Krenke, M. Acet, E. F. Wassermann, X. Moya, L. Mañosa, A. Planes, Ferromagnetism in the austenitic and martensitic states of Ni-Mn-In alloys, Phys. Rev. B 2006, 73, 174413.

[27] E. Dogan, I. Karaman, N. Singh, A. Chivukula, H. S. Thawabi, R. Arroyave, The effect of electronic and magnetic valences on the martensitic transformation of CoNiGa shape memory alloys, Acta Mater. 2012, 60, 3545.

[28] N. Singh, E. Dogan, I. Karaman, R. Arroyave, Effect of configurational order on the magnetic characteristics of Co-Ni-Ga ferromagnetic shape memory alloys, Phys. Rev. B 2011, 84, 184201.

[29] W. Ito, K. Ito, R. Y. Umetsu, R. Kainuma, K. Koyama, K. Watanabe, A. Fujita, K. Oikawa, K. Ishida, T. Kanomata, Kinetic arrest of martensitic transformation in the NiCoMnIn metamagnetic shape memory alloy, Appl. Phys. Lett. 2008, 92, 021908.

[30] W. Ito, M. Nagasako, R. Y. Umetsu, R. Kainuma, T. Kanomata, K. Ishida, Appl. Phys. Lett. 2008, Atomic ordering and magnetic properties in the $\mathrm{Ni}[\mathrm{sub} 45] \mathrm{Co}[\mathrm{sub} 5] \mathrm{Mn}[\mathrm{sub} 36.7] \operatorname{In}[\mathrm{sub}$ 13.3] metamagnetic shape memory alloy, 93, 232503. 
[31] W. Ito, R. Y. Umetsu, R. Kainuma, T. Kakeshita, K. Ishida, Heat-induced and isothermal martensitic transformations from kinetically arrested parent phase in NiCoMnIn metamagnetic shape memory alloy, Scr. Mater. 2010, 63, 73.

[32] H. E. Karaca, I. Karaman, B. Basaran, Y. Ren, Y. I. Chumlyakov, H. J. Maier, Magnetic field-induced phase transformation in NiMnColn magnetic shape-memory alloys-a new actuation mechanism with large work output, Adv. Funct. Mater. 2009, 19, 983.

[33] H. E. Karaca, I. Karaman, A. Brewer, B. Basaran, Y. I. Chumlyakov, H. J. Maier, Shape memory and pseudoelasticity response of NiMnCoIn magnetic shape memory alloy single crystals, Scripta Mater. 2008, 58, 815.

[34] Y.H. Lee, M. Todai, T. Okuyama, T. Fukuda, T. Kakeshita, R. Kainuma, Isothermal nature of martensitic transformation in an Ni45Co5Mn36.5In13.5 magnetic shape memory alloy, Scripta Mater. 2011, 64, 927.

[35] J. A. Monroe, I. Karaman, B. Basaran, W. Ito, R.Y. Umetsu, R. Kainuma, K. Koyama, Y.I. Chumlyakov, Direct Measurement of Large Reversible Magnetic-Field-Induced Strain in Ni-CoMn-In Metamagnetic Shape Memory Alloys, Acta Materialia, 2012, 60, 6883.

[36] X. Xu, W. Ito, I. Katakura, M. Tokunaga, R. Kainuma, In situ optical microscopic observation of NiCoMnIn metamagnetic shape memory alloy under pulsed high magnetic field, Scripta Mater. 2011, 65, 946.

[37] X. Xu, W. Ito, R. Y. Umetsu, R. Kainuma, K. Ishida, Anomaly of critical stress in stressinduced transformation of NiCoMnIn metamagnetic shape memory alloy, Appl. Phys. Lett. 2009, 95 .

[38] L. E. Cross, Relaxor ferroelectrics, Ferroelectrics 1987, 76, 241.

[39] Y. Wang, Y. Zhou, J. Zhang, X. Ding, S. Yang, X. Song, X. Ren, K. Otsuka, Evolution of the relaxation spectrum during the strain glass transition of Ti48.5Ni51.5 alloy, Acta Mater. 2010, 58, 4723.

[40] V. Recarte, J.I. Pérez-Landazábal, V. Sánchez-Alarcos, J.A. Rodríguez-Velamazán, Dependence of the martensitic transformation and magnetic transition on the atomic order in $\mathrm{Ni}-$ Mn-In metamagnetic shape memory alloys, Acta Materialia 2012, 60, 1937.

[41] E. Sasioglu, L. M. Sandratskii, P. Bruno, Role of conduction electrons in mediating exchange interactions in Mn-based Heusler alloys, Phys. Rev. B 2008, 77.

[42] M. A. Ruderman, C. Kittel, Indirect exchange coupling of nuclear magnetic moments by conduction electrons, Phys. Rev. 1954, 96, 99.

[43] T. Graf, C. Felser, S. S. P. Parkin, Simple rules for the understanding of Heusler compounds, Prog. Solid State Ch. 2011, 39, 1. 
[44] R. Y. Umetsu, K. Ito, W. Ito, K. Koyama, T. Kanomata, K. Ishida, R. Kainuma, Kinetic arrest behavior in martensitic transformation of NiCoMnSn metamagnetic shape memory alloy, J. Alloys Compd. 2011, 509, 1389.

[45] S. Kustov, M. L. Corro, J. Pons, E. Cesari, Entropy change and effect of magnetic field on martensitic transformation in a metamagnetic Ni-Co-Mn-In shape memory alloy, App. Phys. Lett. $2009,94$.

[46] T. Fukuda, T. Kakeshita, Y. Lee, An interpretation of the kinetics of martensitic transformation in a Ni45Co5Mn36.5In13.5 alloy, Acta Mater. 2014, 81, 121. 


\section{Figures:}
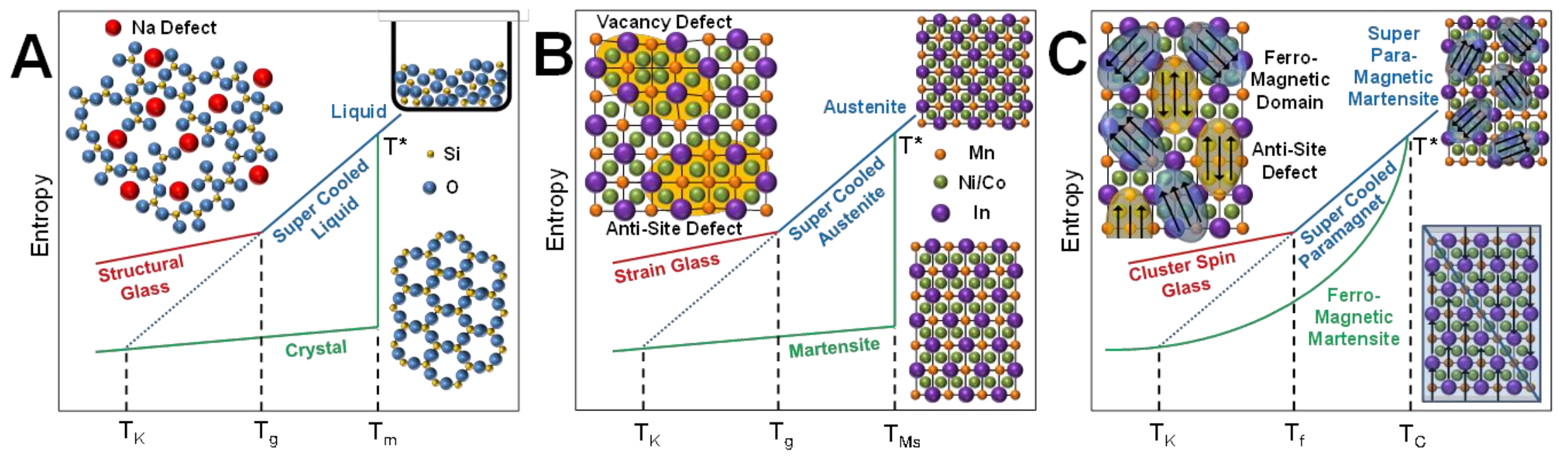

Figure 1. Schematic representations showing similarities between (A) structural glasses, (B) strain glasses and (C) cluster spin glasses. Critical temperatures include melting $\left(\mathrm{T}_{\mathrm{m}}\right)$; martensite start $\left(\mathrm{T}_{\mathrm{Ms}}\right)$ and Curie $\left(\mathrm{T}_{\mathrm{C}}\right)$ phase transitions, super cooling $\left(\mathrm{T}^{*}\right)$, structural and strain glass transitions $(\mathrm{Tg})$, cluster spin glass feezing $\left(\mathrm{T}_{\mathrm{f}}\right)$ and Kauzmann $\left(\mathrm{T}_{\mathrm{K}}\right)$. 


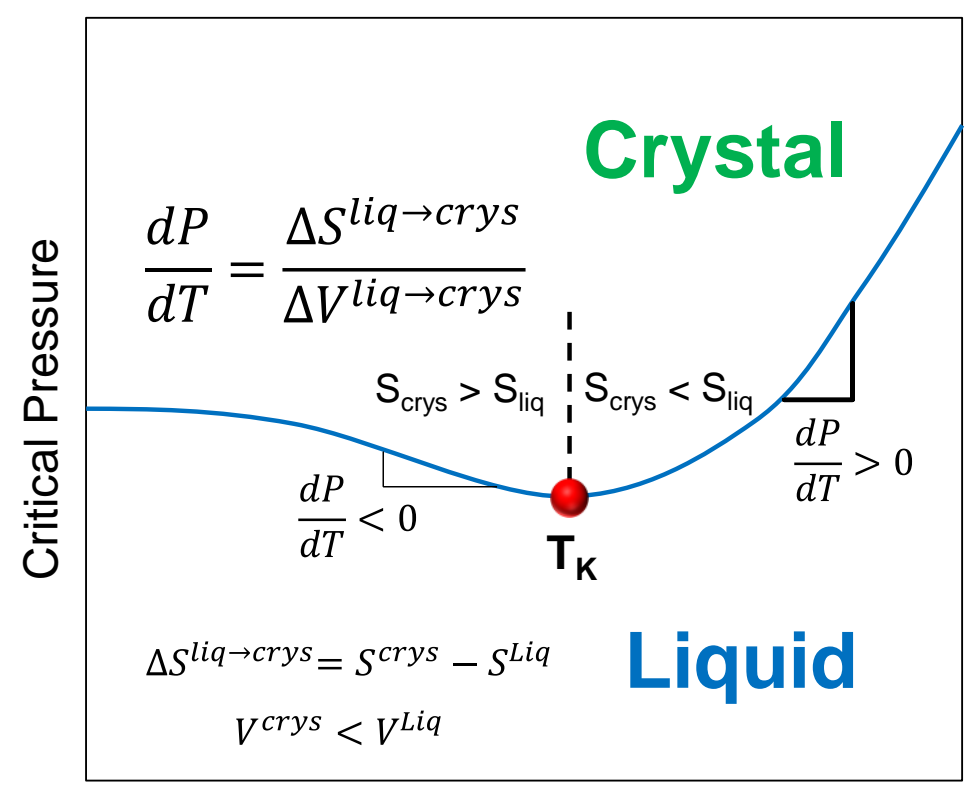

Temperature

Figure 2. A pressure vs temperature schematic for He III or IV displaying the thermodynamic scheme used to determine a Kauzmann point adapted from [20]. 


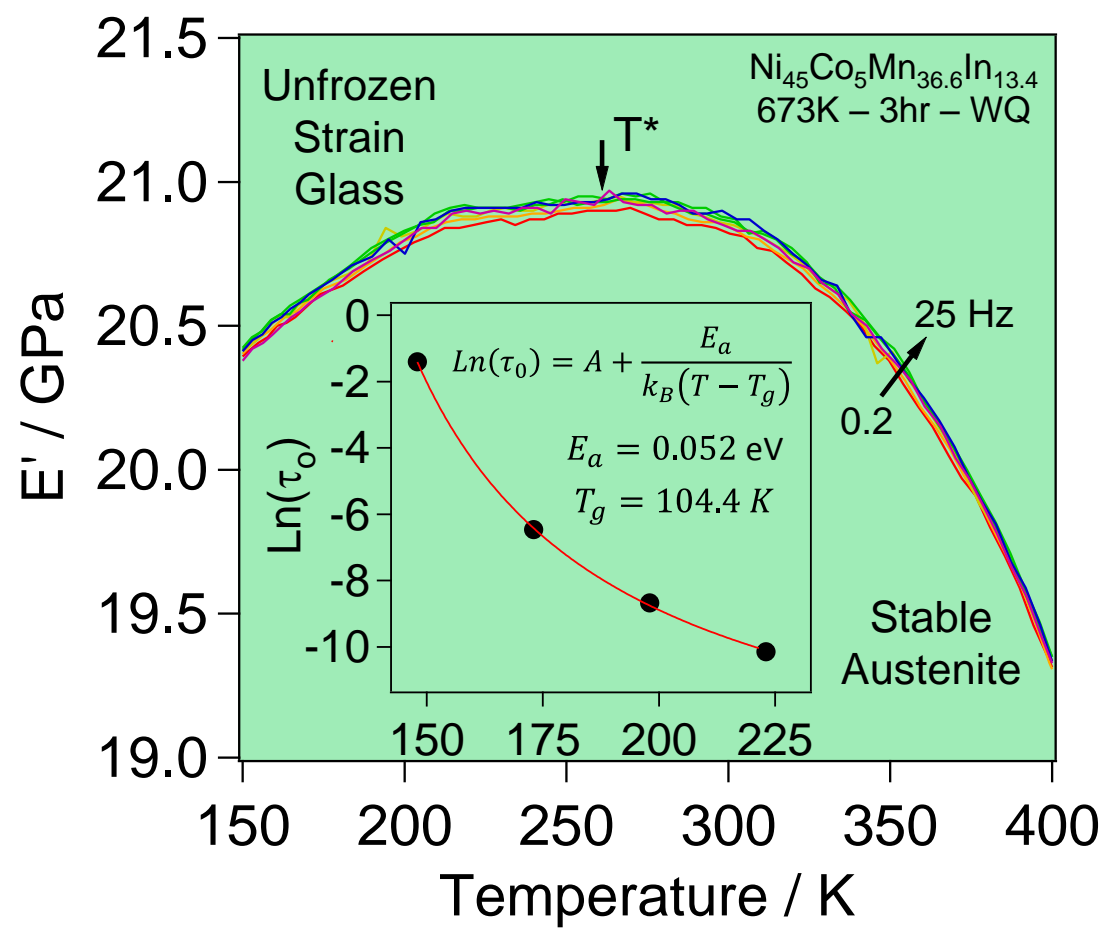

Figure 4. The storage modulus (E') vs temperature showing a frequency dependent second order transition at $\mathrm{T}^{*}=261 \mathrm{~K}$. The inset shows a Vogel-Fulcher-Tamman type relationship in the characteristic relaxation time $\left(\tau_{0}\right)$ obtained from frequency dependent modulus measurements at four temperatures. 

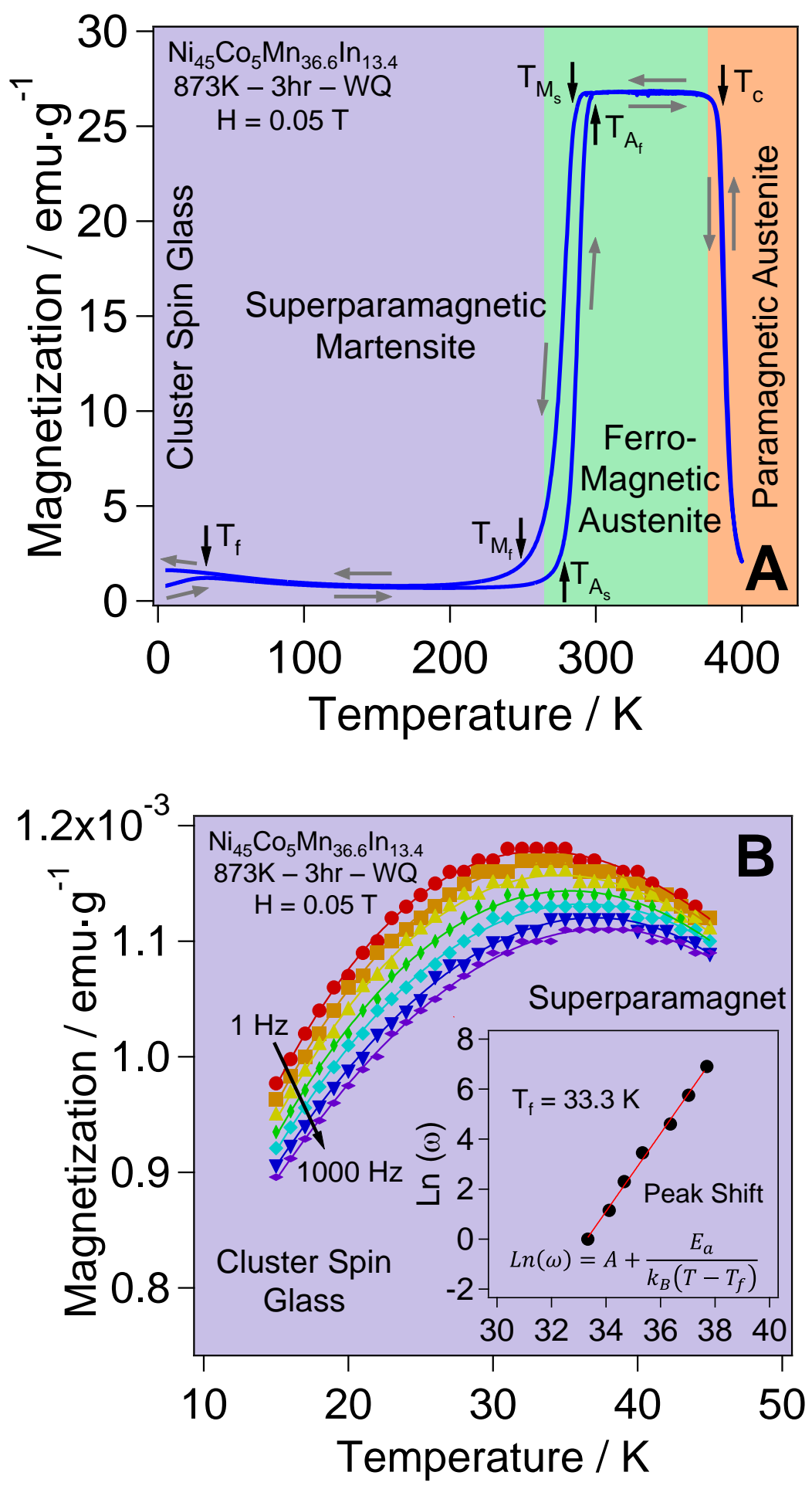

Figure 3. Magnetization vs. temperature experiments for the $873 \mathrm{~K}-3 \mathrm{hr}-\mathrm{WQ}$ sample displaying A) DC field heating after zero-field cooling and field cooling with inflections showing the Currie $\left(T_{C}\right)$, martensitic transformation $\left(T_{M_{s}}, T_{M_{f}}, T_{A_{s}}\right.$ and $\left.T_{A_{f}}\right)$ and path dependent cluster spin glass freezing $\left(T_{f}\right)$ temperatures and $\left.\mathrm{B}\right)$ the real part of the AC magnetization across $T_{f}$ showing a cluster spin glass frequency dependent peak shift between $1 \mathrm{~Hz}$ and $1000 \mathrm{~Hz}$. 


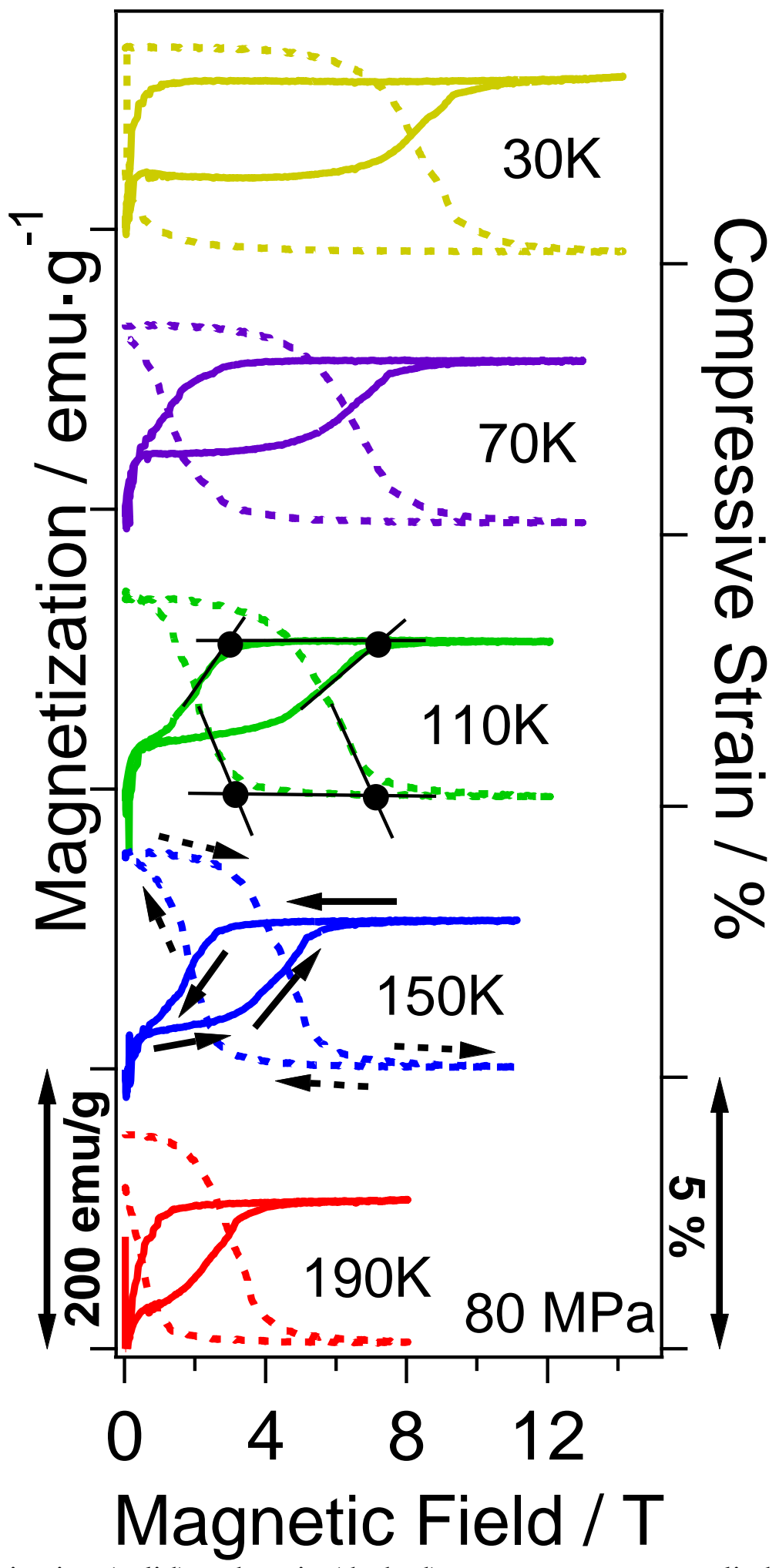

Figure 5. Magnetization (solid) and strain (dashed) measurements vs applied magnetic field for a [100] oriented single crystal in the $673 \mathrm{~K}-3 \mathrm{hr}-$ WQ condition. Critical transformation austenite finish $\left(H_{A_{f}}\right)$ and martensite start $\left(H_{M_{S}}\right)$ magnetic fields are determined using the slope intercept method. 


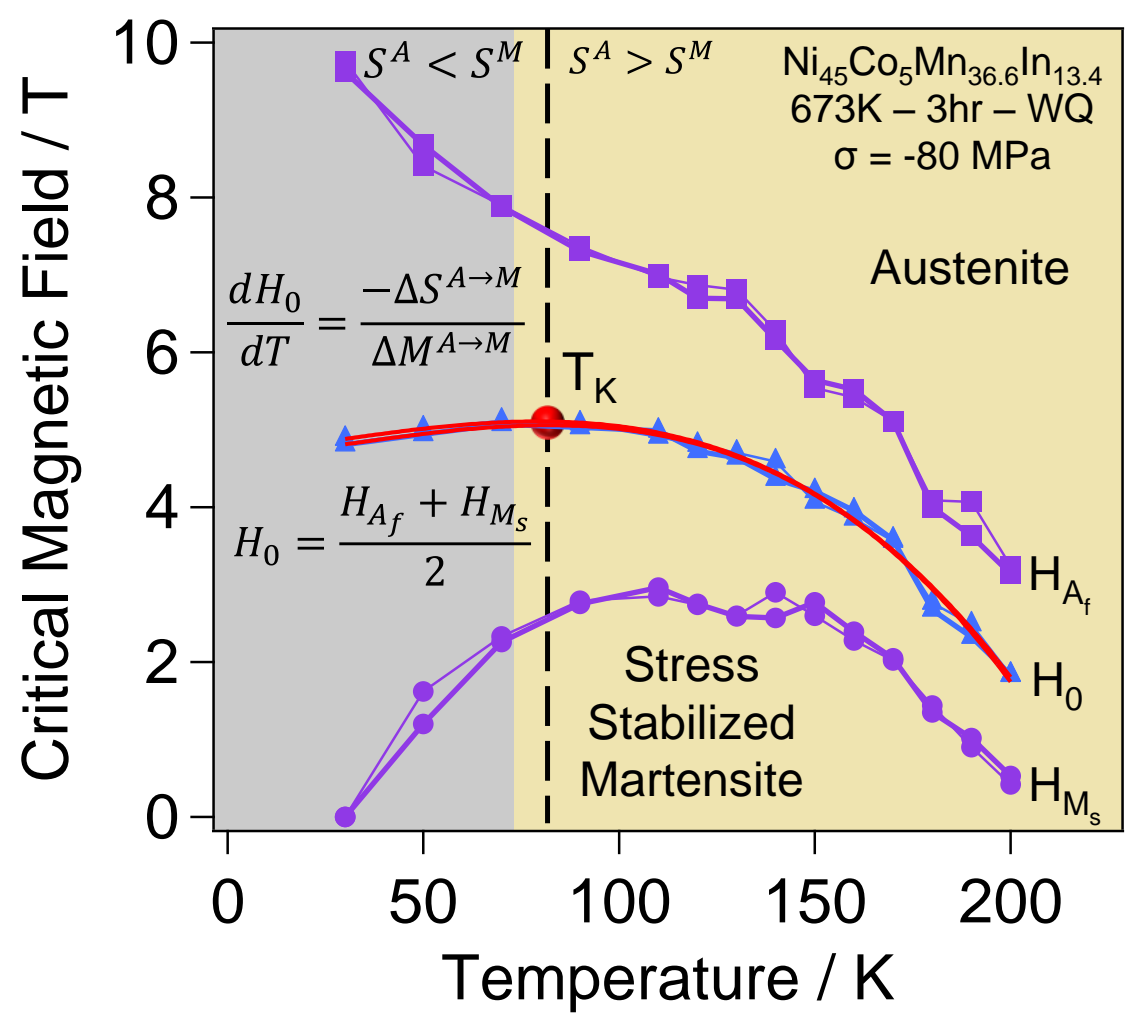

Figure 6. Martensite start $\left(H_{M_{S}}\right)$, austenite finish $\left(H_{A_{f}}\right)$ and equilibrium $\left(H_{0}\right)$ transformation magnetic fields vs. temperature data under $-80 \mathrm{MPa}$ stress that show a Kauzmann point at $\mathrm{T}_{\mathrm{K}}=$ $80 \mathrm{~K}$. Two lines for each transformation magnetic fields are the results from the strain and magnetization measurements shown in Figure 5, the fact that they match quite well, indicating the high fidelity of the measurements. 

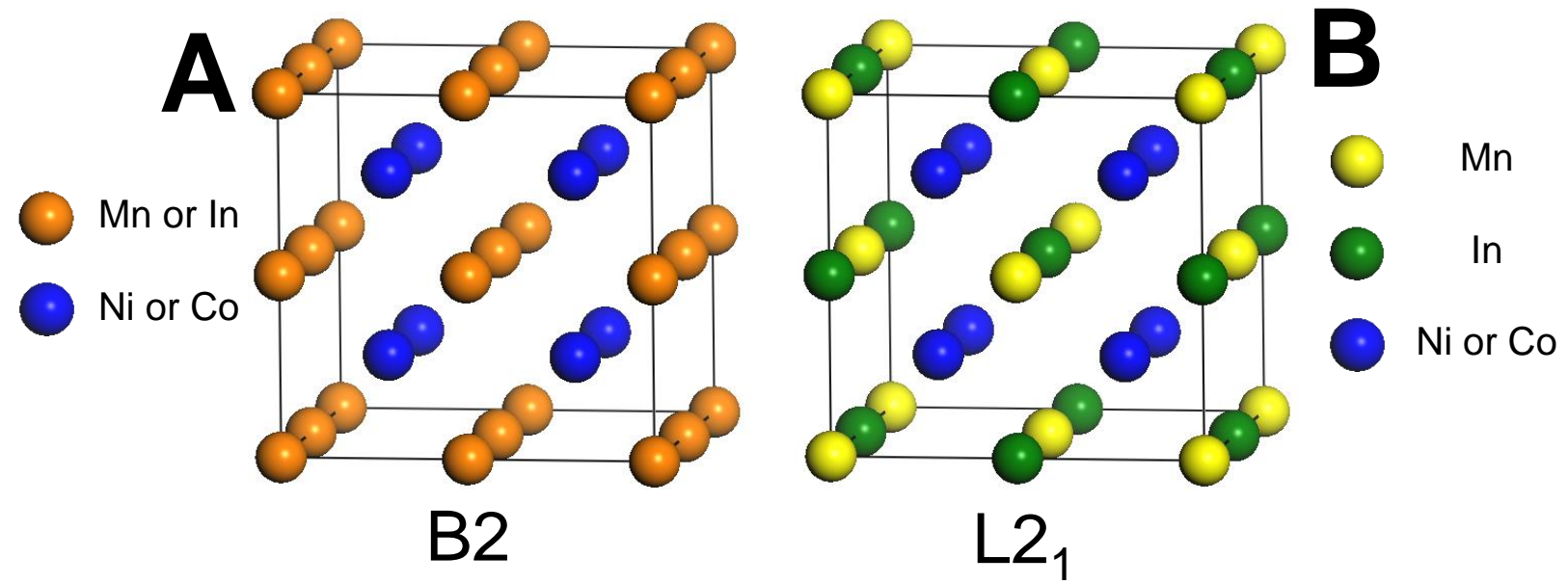

Figure 7. Unit cells for (A) B2 ordering and (B) L $2{ }_{1}$ ordering observed in NiCoMnIn Heusler alloys. 

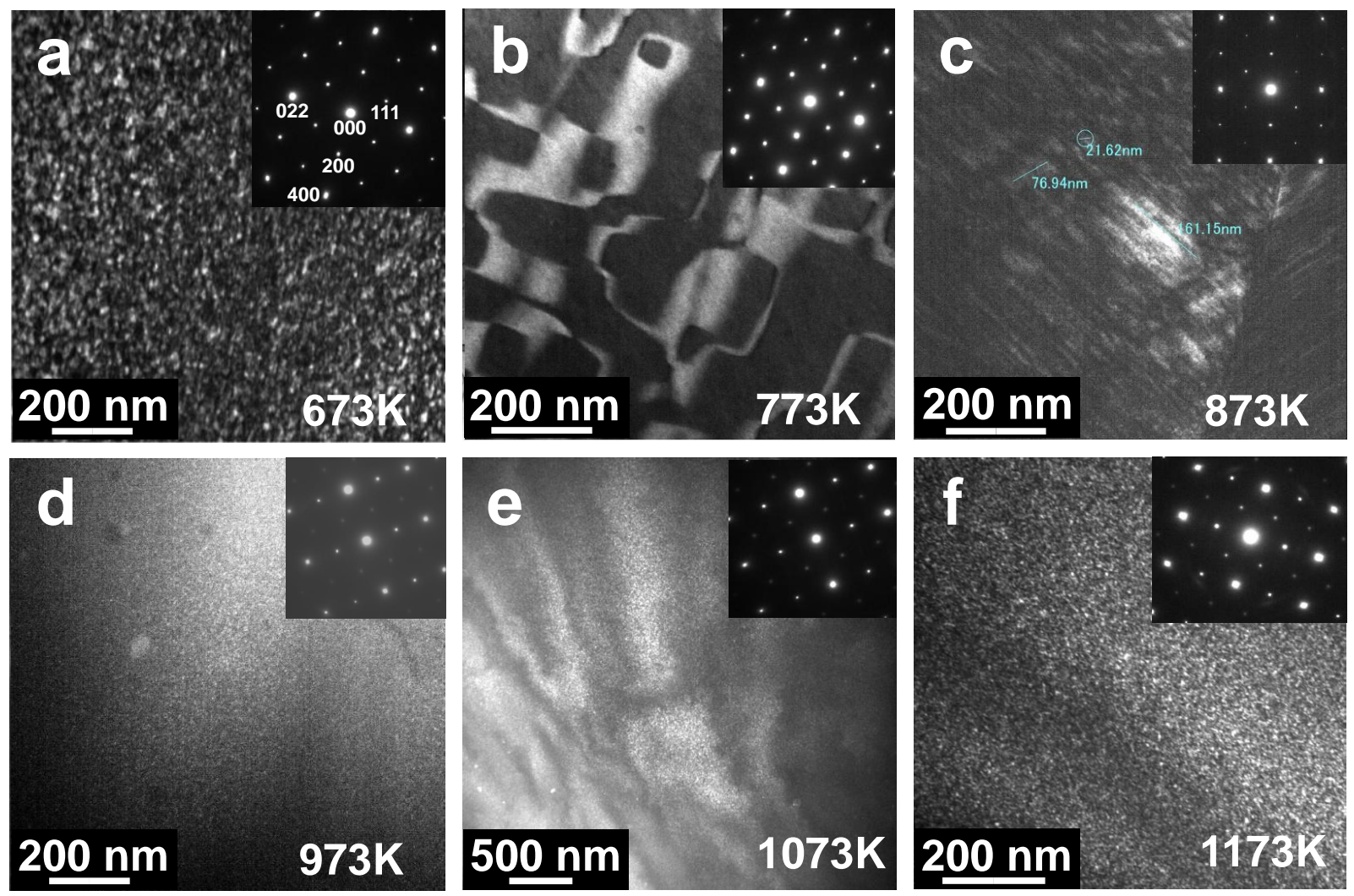

Figure 8. Dark field TEM using the (111) L2 1 superlattice reflection for the $673 \mathrm{~K}, 773 \mathrm{~K}, 873$ $\mathrm{K}, 973 \mathrm{~K}, 1073 \mathrm{~K}$ and $1173 \mathrm{~K}$ heat treated samples. 


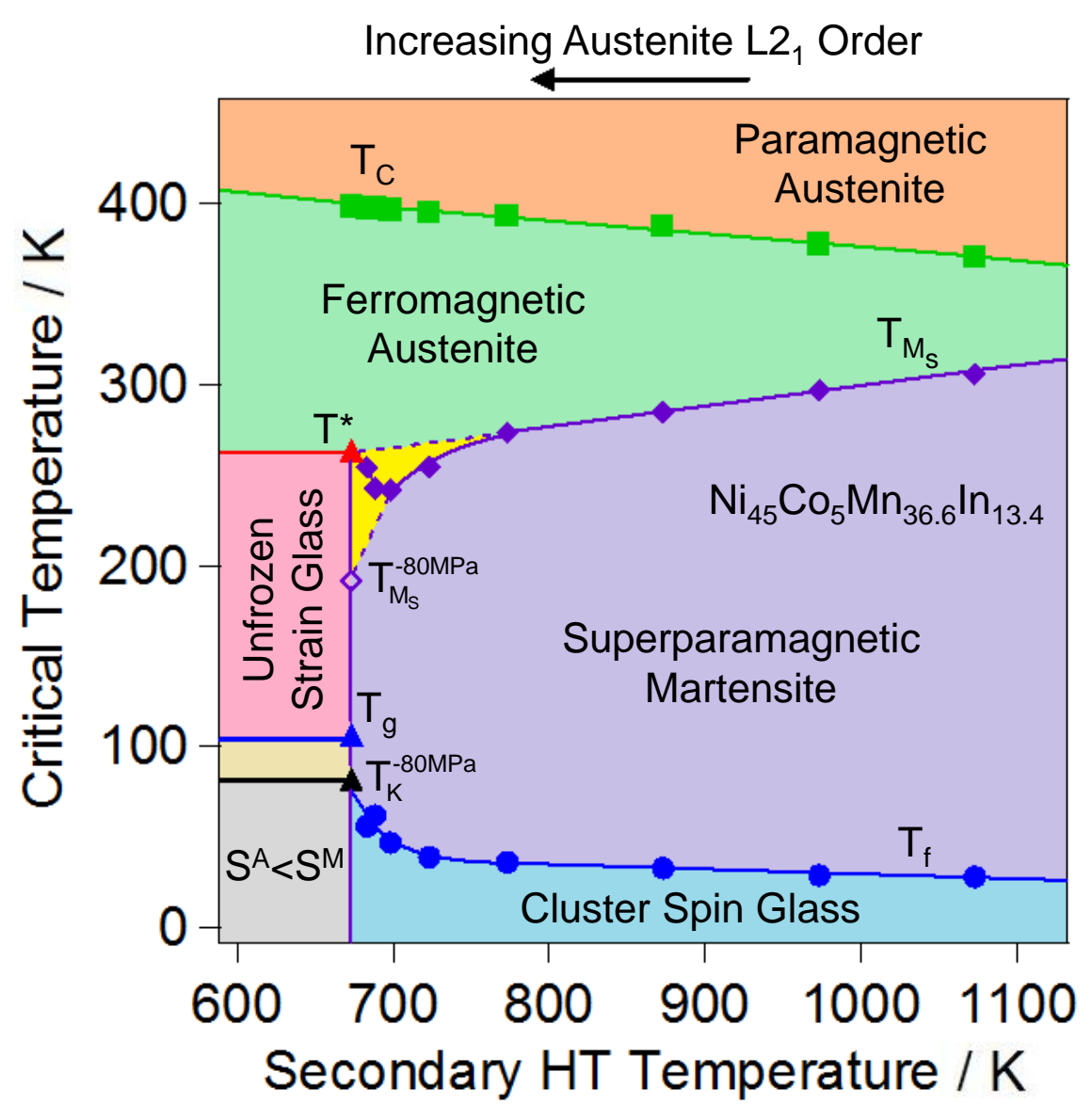

Figure 9. Phase diagram showing the secondary heat treatment influence on the Curie $\left(\mathrm{T}_{\mathrm{C}}\right)$, martensite start $\left(\mathrm{T}_{\mathrm{Ms}}\right)$, spin glass feezing $\left(\mathrm{T}_{\mathrm{f}}\right)$, supercooled austenite $\left(\mathrm{T}^{*}\right)$, strain glass freezing $\left(\mathrm{T}_{\mathrm{g}}\right)$ and Kauzmann $\left(\mathrm{T}_{\mathrm{K}}\right)$ temperatures. 

Supplementary Material
Click here to download S Supplementary Material
Click here to download Supplementary Material: Monroe_Acta MaterialiaFinal2-afterreview-textonly-w-ohighlights.docx

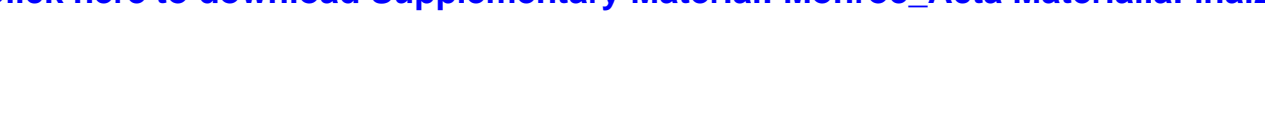

$x$
. (1) . . . . .

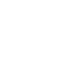

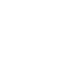
. . . . . . . 
Supplementary Material
Click here to download Supplementary Material: Monroe_Acta MaterialiaFinal2Figures-Afterreview.pdf

Supplementary Material
Click here to download Supplementary Material: Monroe_Acta MaterialiaFinal2Figures-Afterreview.pdf 作

f

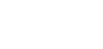

$\sqrt{10}$
$\sqrt{3}$ _Acta Materialiafinal2Figures-Afterreview.pdf (1) (1) (1) (1) . . . (1) (1) (1) . 
